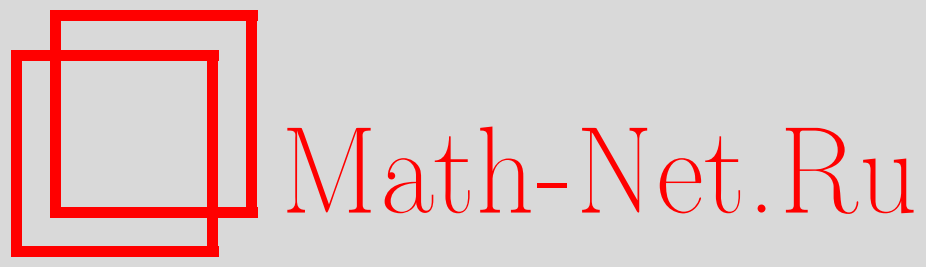

А. М. Вершик, М. И. Граев, Интегральные модели унитарных представлений групп токов со значениями в полупрямых произведениях, Функи. анализ и его прил., 2008, том 42, выпуск 4, 37-49

DOI: https://doi.org/10.4213/faa2929

Использование Общероссийского математического портала MathNet.Ru подразумевает, что вы прочитали и согласны с пользовательским соглашением http://www . mathnet.ru/rus/agreement

Параметры загрузки:

IP : 54.157 .27 .8

26 апреля 2023 г., 18:21:48

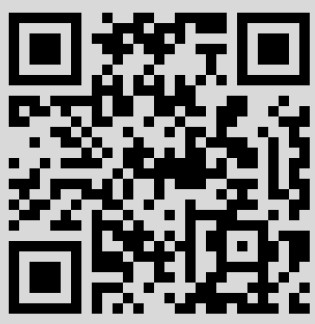


Функиионалъный анализ и его приложения

2008, т. 42, вып. 4, с. 37-49

УДК 517.5

\title{
Интегральные модели унитарных представлений групп токов со значениями в полупрямых произведениях*
}

\author{
(c) 2008. А. М. ВЕРшик, М. И. ГРАЕВ \\ Дорогому Израилю Моисеевичу Гельфанду к его 95-летию
}

В 1972 г. по инициативе И. М. Гельфанда был начат цикл работ трех авторов (самого Израиля Моисеевича, М. И. Граева и А. М. Вершика) об унитарных представлениях функциональных групп или групп токов. Задача, которую поставил И. М. Гельфанд первому автору настоящей работы весной 1972 г. и с которой начался этот цикл работ, состояла в том, чтобы выяснить, существует ли «мультипликативный интеграл представлений» (см. далее) для группы $S L(2, \mathbb{R})$. Вскоре выяснилось, что ответ на этот вопрос положителен, и в первой статье на эту тему [2] было описано несколько моделей этого представления. Основная идея состояла в изучении окрестности единичного представления самой группы $S L(2, \mathbb{R})$ и так называемого особого (бесконечно малого) ее представления, первая группа когомологий которого нетривиальна. Мультипликативный интеграл представлений есть, по выражению авторов, «тензорное произведение бесконечно большого числа бесконечно малых представлений». В дальнейших совместных работах были найдены различные обобщения этой конструкции (на простые группы Ли ранга 1, для групп диффеоморфизмов и пр.) и развита техника работы с такими представлениями. Начиная с 2004 г., авторы настоящей статьи предприняли систематическое изучение интегральной модели представления групп токов, основанной на новой интерпретации континуального тензорного произведения, отличной от фоковской и существенно использующей замечательную сигма-конечную меру в пространстве распределений. Систематическое изложение всей этой области будет содержаться в готовящейся книге трех упомянутых авторов «Теория представлений групп токов».

\section{§1. Введение}

В работе дается краткое изложение теории интегральных моделей представлений групп токов, введенных в предшествующей работе авторов [10]. Интегральные модели для групп токов параболических подгрупп простых групп Ли ранга 1 используются для построения представлений групп токов самих простых групп ранга 1. Мы устанавливаем также связь интегральных моделей и построенных на их основе представлений групп токов простых групп ранга 1 с фоковскими моделями, рассматривавшимися ранее ([5], [6]).

Под группами токов имеются в виду группы измеримых функций на том или ином пространстве или многообразии с мерой со значениями в некоторой груп-

* Исследования первого автора поддержаны грантами НШ 2460.2008.1 и РФФИ 08-0100379. Исследования второго автора поддержаны РФФИ, грант 07-01-00101а. 
пе коэффициентов и с поточечным умножением. Интерес представляют лишь такие неприводимые представления групп токов, которые инвариантны относительно некоторой группы автоморфизмов области задания, например, группы всех преобразований, сохраняющих меру, или группы вращений окружности в случае групп петель и т.д. Такое представление (если оно существует) названо в [2] мультипликативным интегралом представлений, поскольку оно в определенном смысле является непрерывным тензорным произведением представлений группы коэффициентов. Вопрос о существовании таких представлений для данной группы коэффициентов и данной области задания далеко не тривиален. Общая схема изучения представлений групп токов в фоковском пространстве (т.е. в гильбертовом пространстве, снабженном фоковской факторизацией, с которой согласовано представление токов) дана в работе [1]. В частности, база группы токов в этом случае произвольна, а группа коэффициентов должна обладать неприводимым унитарным представлением с нетривиальной группой первых когомологий. Последнее, как показано в [13], возможно только в том случае, когда единичное представление группы коэффициентов неизолированно в множестве всех неприводимых унитарных представлений в топологии Фелла (см. [18]). В современных терминах это означает, что группа не должна обладать свойством (Т) Каждана. Неприводимое унитарное представление $\pi$ с нетривиальной группой $H^{1}(G ; \pi)$ называется особъм. Среди простых групп Ли особое представление существует лишь у некоторых групп ранга 1, а именно у групп движений вещественного и комплексного пространств Лобачевского $S U(n, 1)$ и $S O(n, 1)$. Для групп токов с такими коэффициентами соответствующие неприводимые представления были впервые построены в работах [2], [3]. Пространством этих представлений служило фоковское пространство, являющееся экспонентой одночастичного пространства, которое, в свою очередь, есть прямой интеграл особых представлений. Конструкция представления группы токов в фоковском пространстве существенно использует нетривиальный 1-коцикл группы коэффициентов в ее особом представлении. Фоковская модель удобна и используется в большинстве работ по теории представлений бесконечномерных групп. В этой модели естественным образом реализуются операторы, отвечающие элементам максимальной компактной подгруппы группы токов. Заметим еще, что структура фоковского гильбертова пространства эквивалентным образом описывается с помощью бесконечномерной гауссовой меры («белого шума») и что предлагаемая далее модель использует другую замечательную меру в бесконечномерном пространстве.

Изучение другой модели представления группы токов, в которой диагонализуются операторы, отвечающие элементам унипотентной подгруппы, началось с работы [5]. Этот подход постепенно привел к совершенно новому пониманию природы мультипликативных интегралов представлений и непрерывных тензорных произведений. В его основе лежат два принципиальных соображения.

1) Как и для классических простых групп Ли, например для $S L(2, \mathbb{R})$, построение представления группы токов простых групп Ли следует начинать с построения представления группь токов максимальной параболической подгрynnы, а затем продолжать это представление на всю группу токов простой группы. 
2) В свою очередь, существование представления группы токов максимальной параболической подгруппы простой группы Ли ранга 1 вытекает из того обстоятельства, что на конусе дискретных конечных положительных мер на многообразии, инвариантных относительно непрерывного аналога картановской nодгрупnы - группы мультипликаторов с конечным интегралом от логарифма, - имеется замечательная сигма-конечная мера. Эта мера, обнаруженная в работе [5], изучавшаяся в статьях [15], [14] и аксиоматизированная и подробно исследованная в недавних работах [11], [12], названа бесконечномерной мерой Лебега. Она позволяет кардинально упростить теорию представлений групп токов полупрямых произведений, но, кроме того, она оказывается тесно связанной с многочисленными комбинаторными, аналитическими и вероятностными задачами.

По причине, которая станет ясной из конструкции, мы называем этот тип моделей представлений групп токов интегральными моделями. В этой работе мы даем последовательное изложение этой альтернативной конструкции - интегральных моделей представлений группы токов полупрямых произведений - и показываем, как продолжить их на группу токов групп движений вещественного и комплексного пространств Лобачевского.

В коротком изложении наша модель непрерывного тензорного произведения гильбертовых пространств и представлений групп токов в этих пространствах трактуется следующим образом:

а) рассматривается пространство счетных линейных положительных комбинаций дельта-мер на базе со сходящейся суммой коэффициентов («пространство траекторий»), которое снабжается бесконечномерной лебеговой мерой, определяемой далее ([12], [11]);

b) каждой траектории сопоставляется счетное тензорное произведение гильбертовых пространств, взятых по дельта-мерам вдоль траектории, в каждом из которых определено представление подгруппы с параметром, равным коэффициенту при дельта-мере;

с) определяется прямой интеграл по лебеговой мере на пространстве траекторий от построенных счетных тензорных произведений.

Конструкция дает представления групп токов (и, в частности, групп петель) в гильбертовом пространстве с дополнительной структурой, обозначаемом через INT; эти представления эквивалентны представлениям, построенным ранее в [2], [3] и реализованным в фоковском пространстве ЕХР. Изоморфизм (единственный с точностью до скаляра) между пространствами EXP и INT представляет интерес сам по себе ${ }^{1)}$. Выигрыш от такого способа построения представлений группы токов состоит еще и в том, что неприводимость представления группы токов параболической подгруппы становится почти очевидной, равно как и анализ особого представления; вопрос лишь в том, чтобы доопределить представления на группу токов всей простой группы. Это оказывается возможным для $O(n, 1)$ и $U(n, 1)$ и невозможно для $S p(n, 1)$.

В $§ 2$ мы определяем класс полупрямых произведений, служащих в дальнейшем группами коэффициентов, для которых далее определяется конструкция

1) Заметим, что этот изоморфизм сохраняет структуру фоковской факторизации тензорных произведений гильбертовых пространств. В то же время существуют нефоковские факторизации в гильбертовых пространствах (см. [17]) и, следовательно, открыта возможность строить представления групп токов с факторизацией, не изоморфной фоковской. Насколько известно, эта возможность не исследована до сих пор. 
интегральных моделей представлений групп токов. Мы вводим понятие канонического представления, с помощью которого определяется особое представление полупрямого произведения. В следующем параграфе приведена основная конструкция. В $\S 4$ содержатся формулы для сферической функции интегральной модели и устанавливается изоморфизм этой модели с фоковской. В 5 детально описаны интегральные модели для простейшего случая - параболической подгруппы группы $S L(2, \mathbb{R})$ - и построено продолжение этих интегральных моделей до представлений всей группы токов $S L(2, \mathbb{R})^{X}$. Параграф 6 посвящен интегральным моделям для общего случая максимальных параболических подгрупп $P$ групп $O(n, 1)$ и $U(n, 1)$. Мы формулируем результаты о продолжении этих интегральных моделей до представлений соответственно групп $O(n, 1)^{X}$ и $U(n, 1)^{X}$, а для случая $P \subset U(n, 1)$ приводим явное описание самих интегральных моделей и их продолжений до представлений группы $U(n, 1)^{X}$. Здесь различаются случаи ортогонального и унитарного особых представлений: в унитарном случае возникают проективные унитарные представления группы $U(n, 1)^{X}$, рассмотренные также в [6]. Существует связь между этими проективными представлениями и построенными в [4] унитарными (непроективными) представлениями группы токов $\widetilde{G}^{X}$, где $\widetilde{G}$ - универсальная накрывающая группы $U(n, 1)$.

Доказательства и подробная версия теории интегральных моделей излагаются в готовящейся статье авторов.

\section{§2. Исходная конструкция: особые представления полупрямых произведений групп}

Начнем с общей схемы, применимой, в частности, к параболическим подгруппам простых групп ранга 1. Рассмотрим полупрямое произведение $P=S \wedge P_{0}$ локально компактной группы $P_{0}$ и ее мультипликативной однопараметрической группы автоморфизмов $S \cong \mathbb{R}_{+}^{*}$. Будем обозначать через $g^{r}$ образ элемента $g \in P_{0}$ под действием автоморфизма $r \in S$. В дальнейшем термин «представление» означает, если не оговорено противное, ортогональное или унитарное представление. Указанная группа автоморфизмов действует и на представлениях $T$ группы $P_{0}$, а именно, с каждым представлением $T$ подгруппы $P_{0}$ в некотором гильбертовом пространстве $H$ связано семейство сопряженных с $T$ представлений $T_{r}, r \in \mathbb{R}_{+}^{*}$, полученных из $T$ с помощью автоморфизмов из группы $\mathbb{R}_{+}^{*}: T_{r}(g) \equiv T\left(g^{r}\right)$.

Циклическое представление $T$ подгруппы $P_{0}$ называется каноническим относительно группы автоморфизмов $S$, если существует такой циклический вектор $h \in H$ единичной нормы, что $\left\|T_{r}(g) h-h\right\|<c(g) r$ при достаточно малых $r$ и для любого $g \in P_{0}$. Вектор $h$ с указанным свойством называется почти инвариантным относительно представления $T$. Очевидно, что в топологии Фелла на пространстве представлений (см., например, [18]) представления $T_{r}$ стремятся при $r \rightarrow 0$ к единичному представлению. В терминах матричных элементов представления $T$ (состояний), отвечающих циклическим элементам $h$, наше определение выглядит так:

$$
\lim _{r \rightarrow 0} \frac{\varphi_{h}\left(g^{r}\right)-1}{r}=-\left.\frac{d \varphi_{h}\left(g^{r}\right)}{d r}\right|_{r=0} \equiv-\psi(g)<\infty
$$


для всех $g \in G$, где $\varphi_{h}(g)=\operatorname{Re}(\langle T(g) h, h\rangle)$ есть нормированная положительно определенная функция на группе $G$ («состояние»).1)

$\mathrm{C}$ каждым каноническим представлением $T$ подгруппы $P_{0}$ связано представление $\widetilde{T}$ группы $P=\mathbb{R}_{+}^{*} \wedge P_{0}$ в прямом интеграле

$$
\mathscr{H}=\int_{0}^{\infty} H_{r} r^{-1} d r
$$

гильбертовых пространств $H_{r} \equiv H$ по мультипликативной мере Хаара $r^{-1} d r$ на $\mathbb{R}_{+}^{*}$, т.е. в пространстве сечений $f(r)$ расслоения над $\mathbb{R}_{+}^{*}$ со слоем $H_{r}$ над $r \in \mathbb{R}_{+}^{*}$. Операторы $\widetilde{T}\left(g_{0}\right), g_{0} \in P_{0}$, действуют на $\mathscr{H}$ послойно, $\left(\widetilde{T}\left(g_{0}\right) f\right)(r)=$ $T_{r}\left(g_{0}\right) f(r)$, а операторы $\widetilde{T}\left(r_{0}\right), r_{0} \in \mathbb{R}_{+}^{*}$, переставляют слои: $\left(\widetilde{T}\left(r_{0}\right) f\right)(r)=$ $f\left(r_{0} r\right)$.

Лемма. Представление $\widetilde{T}$ группы $P$ является особым (см. [8]).

Действительно, по построению оно обладает нетривиальными 1-коциклами $b: P \rightarrow \mathscr{H}$ вида

$$
b(g, r)=\left(\widetilde{T}(g) f_{0}\right)(r)-f_{0}(r), \quad f_{0}(r)=\varphi(r) h_{r},
$$

где $h_{r}=h$ - почти инвариантный относительно $T$ вектор в $H$, а $\varphi(r)-$ любая гладкая положительная функция на $[0, \infty)$, такая, что $\int_{\epsilon}^{\infty} \varphi^{2}(r) r^{-1} d r<\infty$ при $\epsilon>0$ и $\varphi(0) \neq 0$.

Отметим, что 1-коциклы, отвечающие функциям $\varphi$ и $\varphi_{1}$, эквивалентны тогда и только тогда, когда $\varphi(0)=\varphi_{1}(0)$.

Примерами групп $P=\mathbb{R}_{+}^{*} \curlywedge P_{0}$, подгруппы $P_{0}$ которых обладают каноническими представлениями, являются максимальные параболические подгруппы групп $O(n, 1), U(n, 1)$ и $S p(n, 1)$. В случае $O(n, 1)$ имеется, с точностью до перехода к сопряженным представлениям, только одно, а в случаях $U(n, 1)$ и $S p(n, 1)$ - счетный набор канонических неприводимых представлений подгруппы $P_{0} \cdot \mathrm{C}$ каждым из этих канонических представлений подгруппы $P_{0}$ связано особое неприводимое представление группы $P$. Отметим, что из всех особых представлений группы $P \subset U(n, 1)$ только два унитарных и одно ортогональное продолжаются до особого представления всей группы $U(n, 1) ;$ у группы $S p(n, 1)$ особых представлений вообще не существует.

\section{§3. Построение интегральной модели представлений (INT)}

Группой токов $P^{X}$, где $P$ - локально компактная группа, а $X$ - пространство с вероятностной мерой $m$, называется группа ограниченных измеримых отображений $X \rightarrow P$ с поточечным умножением. В [10] для групп $P$ вида $P=\mathbb{R}_{+}^{*} \wedge P_{0}$ по каждому каноническому представлению $T$ подгруппы $P_{0}$ в пространстве $H$ с почти инвариантным относительно $T$ вектором $h \in H$ было определено и названо интегралъной моделъю представление группы токов $P^{X}$. По

1) Функция $\psi(\cdot)$, определенная выше, является неограниченной условно положительно определенной функцией и равна квадрату нормы нетривиального коцикла в особом представлении (см. далее). По поводу терминологии заметим, что в работе [10] каноническое представление было названо суммируемым, а в статье [2] каноническими для группы $P S L(2, \mathbb{R})$ названы состояния, инвариантные относительно компактной подгруппы и удовлетворяющие близкому условию. Здесь мы несколько расширяем понятие канонического состояния, используя тот же термин. 
аналогии с фоковскими моделями $\mathrm{EXP} \mathrm{(см.} \mathrm{[5])} \mathrm{будем} \mathrm{обозначать} \mathrm{это} \mathrm{представ-}$ ление группы $P^{X}$ через INT $T$, а через INT $H$ или, подробнее, через $\operatorname{INT}(H, h)-$ гильбертово пространство, в котором оно реализовано.

Конструкция представления INT $T$ группы токов $P^{X}$, где $P=\mathbb{R}_{+}^{*} \wedge P_{0}$, аналогична конструкции особого представления группы $P$ по каноническому представлению $T$ ее подгруппы $P_{0}$. Прежде всего, в этой конструкции пространство $\mathbb{R}_{+}^{*}$ заменено конусом вещественных положительных конечных атомических мер на пространстве $(X, m)$

$$
l_{+}^{1}(X)=\left\{\xi=\sum_{k=1}^{\infty} r_{k} \delta_{x_{k}} \mid r_{k}>0, \sum_{k} r_{k}<\infty\right\}
$$

(его элементы далее обозначаются через $\xi=\left\{r_{k}, x_{k}\right\}$ ). Вместо мультипликативной хааровской меры $r^{-1} d r$ на $\mathbb{R}_{+}^{*}$ мы рассматриваем на $l_{+}^{1}(X)$ бесконечную сигма-конечную меру dLL $(\xi)$. Эта мера (см. [11]), являющаяся бесконечномерным аналогом меры Лебега на октантах $\mathbb{R}_{+}^{n}$, однозначно определяется своим преобразованием Лапласа:

$$
\Psi_{\mathscr{L}}(f) \equiv \int_{l_{+}^{1}(X)} \exp \left(-\sum_{k} r_{k} f\left(x_{k}\right)\right) d \mathscr{L}(\xi)=\exp \left(-\int_{X} \log f(x) d m(x)\right),
$$

где $f$ - произвольная неотрицательная измеримая функция на $(X, m)$ с конечным интегралом от логарифма. Отметим, что, как и в случае классического преобразования Лапласа, равенство (2) сохраняет смысл для комплекснозначных функций $f$ с неотрицательной вещественной частью.

Для интегральной модели существенны лишь два свойства меры $\mathscr{L}$ - ее проективная инвариантность относительно группы мультипликаторов $M_{a}$ (т. е. условие $d \mathscr{L}\left(M_{a} \xi\right)=\varphi(a) d \mathscr{L}(\xi)$, где $\left.\varphi(a)=e^{\frac{1}{2} \int_{X} \log a(x) d m(x)}\right)$ и эргодичность относительно этой группы. Инвариантность следует непосредственно из формулы для преобразования Лапласа; по поводу эргодичности см. [12]. В частности, из проективной инвариантности меры $\mathscr{L}$ следует ортогональность (унитарность) представления INT T, а из эргодичности - его неприводимость. Подробное исследование свойств этой замечательной меры см. в [11], [12].

Далее, в конструкции интегральной модели пространства $H_{r}, r \in \mathbb{R}_{+}^{*}$, с заданными на них представлениями $T_{r}$ подгруппы $P_{0}$ заменены счетными тензорными произведениями $H_{\xi}=\bigotimes_{k=1}^{\infty} H_{r_{k}}, \xi \in l_{+}^{1}(X)$, со стабилизирующим почти инвариантным вектором $h \in H$, на которых действуют представления $T_{\xi}$ группы $P_{0}^{X}$.

По определению счетное тензорное произведение $H_{\xi}=\bigotimes_{k=1}^{\infty} H_{r_{k}}$ со стабилизирующим почти инвариантным вектором $h \in H$ есть пополнение индуктивного предела пространств $\bigotimes_{k=1}^{n} H_{r_{k}}$ относительно вложений $x \in \bigotimes_{k=1}^{n} H_{r_{k}} \mapsto$ $x \otimes h_{r_{n+1}} \in \bigotimes_{k=1}^{n+1} H_{r_{k}}, h_{r_{n+1}}=h$. Операторы представления группы $P_{0}^{X}$ действуют на $H_{\xi}$ по формуле

$$
T_{\xi}(g(\cdot))\left(\bigotimes_{k=1}^{\infty} f_{k}\right)=\bigotimes_{k=1}^{\infty}\left(T_{r_{k}}\left(g\left(x_{k}\right)\right) f_{k}\right)
$$

Отметим, что определение представлений $T_{\xi}$ корректно только для канонических представлений $T$ подгруппы $P_{0}$. 
Пространство INT $H$ интегральной модели $U=\operatorname{INT} T$ представления $T$ группы $P^{X}$ определяется, по аналогии с пространством $\mathscr{H}=\int_{0}^{\infty} H_{r} r^{-1} d r$ представления группы $P$, ассоциированного с каноническим представлением $T$ подгруппы $P_{0}$, как прямой интеграл по мере $\mathscr{L}$ гильбертовых пространств $H_{\xi}$,

$$
\text { INT } H=\int_{l_{+}^{1}(X)}^{\oplus} H_{\xi} d \mathscr{L}(\xi), \quad H_{\xi}=\bigotimes_{k=1}^{\infty} H_{r_{k}},
$$

т. е. как пространство сечений $F(\xi)=F\left(\left\{r_{k}, x_{k}\right\}\right)$ расслоения над $l_{+}^{1}(X)$ со слоем $H_{\xi}$ над каждой точкой $\xi=\left\{r_{k}, x_{k}\right\}$. Действие подгруппы $P_{0}^{X}$ на пространствах $H_{\xi}$ индуцирует ее представление $U=\operatorname{INT} T$ на всем пространстве INT $H$ :

$$
\operatorname{INT} T=\int_{l_{+}^{1}(X)}^{\oplus} T_{\xi} d \mathscr{L}(\xi), \quad \text { где } T_{\xi}=\bigotimes_{k=1}^{\infty} T_{r_{k}, x_{k}} ;
$$

подробнее: $(U(g(\cdot)) F)(\xi)=T_{\xi}(g(\cdot)) F(\xi)$. Продолжение этого представления подгруппы $P_{0}^{X}$ на всю группу $P^{X}$ задается формулой

$$
\left(U\left(r_{0}(\cdot)\right) F\right)(\xi)=e^{\frac{1}{2} \int_{X} \log r_{0}(x) d m(x)} F\left(r_{0}(\cdot) \xi\right) \quad \text { при } r_{0} \in\left(\mathbb{R}_{+}^{*}\right)^{X} .
$$

Из определения следует, что представление INT $T$ группы $P^{X}$ инвариантно относительно преобразований пространства $X$, сохраняющих меру $m$ на $X$.

Теорема 1 [10]. Если каноническое представление $T$ подгруппы $P_{0}$ неприводимо, а представления $T_{r}$ попарно неэквивалентны, то ассоциированное с $T$ представление $\widetilde{T}$ группы $P$ и соответствующее ему представление $U=\operatorname{INT} T$ группы токов $P^{X}$ неприводимы.

Замечание. Конструкция интегральной модели сохраняет смысл при замене вероятностной меры $m$ на $X$ любой положительной конечной мерой $(m(X)=\theta)$ или, что эквивалентно, при добавлении коэффициента $\theta>0$ под знаком ехр в определении преобразования Лапласа меры $\mathscr{L}$. Определенное так однопараметрическое семейство представлений группы $P^{X}$ по-прежнему удовлетворяет утверждению теоремы 1. Для простоты мы ограничиваемся здесь только случаем $\theta=1$.

Определим сферическую функцию представления $U=\operatorname{INT} T$ группы $P^{X}$, ассоциированного с каноническим представлением $T$ подгруппы $P_{0}$ в пространстве $H$, как функцию

$$
\Psi(g)=\langle U(g) \Omega, \Omega\rangle
$$

на $P^{X}$, где $\Omega$ - единичный вектор в пространстве $\widetilde{H}=\mathrm{INT} H$ вида

$$
\Omega(\xi)=\bigotimes_{k=1}^{\infty}\left(e^{-r_{k} / 2} h_{r_{k}}\right), \quad h_{r_{k}}=h \quad \text { при } \xi=\left\{r_{k}, x_{k}\right\} \in l_{+}^{1}(X) .
$$

Теорема 2. Сферическая функиия представления $U$ имеет вид

$$
\Psi(g)=\exp \left(\int_{X}\left(i \operatorname{Im}\left\langle b(g(x)), f_{0}\right\rangle-\frac{1}{2}\|b(g(x))\|^{2}\right) d m(x)\right),
$$

где $f_{0}(r)=e^{-r / 2} h_{r}$ и $b(g)=\left(\widetilde{T}(g) f_{0}\right)(r)-f_{0}(r)$ есть 1-коиикл представления $\widetilde{T}$ группы $P$, ассоииированного с $T, c \mathcal{M} .(1)$. 


\section{§4. Связь между интегральными моделями и фоковскими представлениями}

Для любой локально компактной группы $G$ каждой паре $(\widetilde{T}, b)$, где $\widetilde{T}-$ особое представление группы $G$ в пространстве $\mathscr{H}$, a $b: G \rightarrow \mathscr{H}$ - нетривиальный 1-коцикл, отвечает фоковская модель представления группы токов $G^{X}$, см. [5]. Она реализуется в гильбертовом пространстве $\mathrm{EXP} \mathscr{H}^{X}=\bigoplus_{k=0}^{\infty} S^{k} \mathscr{H}^{X}$, где $S^{k}$ есть $k$-я симметризованная тензорная степень и $\mathscr{H}^{X}=\int_{X}^{\oplus} \mathscr{H}_{x} d m(x)$, $\mathscr{H}_{x}=\mathscr{H}$. Операторы представления задаются на тотальном подмножестве векторов $\operatorname{EXP} v=\mathbb{I} \oplus v \oplus \frac{1}{\sqrt{2 !}} v \otimes v \oplus \frac{1}{\sqrt{3 !}} v \otimes v \otimes v \oplus \cdots$ формулой

$$
U(g) \operatorname{EXP} v=\exp \left(-\frac{1}{2}\left\|b^{X}(g)\right\|^{2}-\left(\widetilde{T}^{X}(g) v, b^{X}(g)\right)\right) \operatorname{EXP}\left(\widetilde{T}^{X}(g) v+b^{X}(g)\right) .
$$

Здесь $\widetilde{T}^{X}$ и $b^{X}$ - представление группы $G^{X}$ в пространстве $\mathscr{H}^{X}$ и 1 -коцикл этой группы со значениями в этом пространстве, порожденные соответственно представлением $\widetilde{T}$ группы $G$ и 1 -коциклом $b$ этой группы в пространстве $\mathscr{H}$. Операторы $U\left(g_{1} g_{2}\right)$ и $U\left(g_{1}\right) U\left(g_{2}\right)$ фоковского представления связаны соотношением $U\left(g_{1} g_{2}\right)=\exp \left(i \operatorname{Im} \int_{X} a\left(g_{1}(x), g_{2}(x)\right) d m(x)\right) U\left(g_{1}\right) U\left(g_{2}\right)$, где $a\left(g_{1}, g_{2}\right)=$ $\left(\widetilde{T}\left(g_{1}\right) b\left(g_{2}\right), b\left(g_{1}\right)\right)$. Таким образом, в случае унитарных представлений $\widetilde{T}$ фоковские представления, вообще говоря, проективны. Однако для групп вида $P=\mathbb{R}_{+}^{*} \wedge P_{0} 2$-коцикл $a\left(g_{1}, g_{2}\right)$ тривиален, а потому эти представления проективно эквивалентны обычным (непроективным) представлениям.

Теорема 3. Пусть $T$ - каноническое представление группы $P_{0}$, удовлетворяющее условиям теоремы 1, и $\widetilde{T}$ - ассоииированное с ним особое представление группь $P$ (см. выше); тогда представление INT $T$ группы $P^{X}$ проективно эквивалентно фоковскому представлению, порожденному тем же представлением Т и 1-коциклом вида (1), в котором $f_{0}(r)=e^{-r / 2} h_{r}$.

\section{§5. Описание интегральных моделей подгруппы $P \subset S L(2, \mathbb{R})$ треугольных матриц и их продолжений на группу $S L(2, \mathbb{R})^{X}$}

Условимся элементы подгруппы $P \subset S L(2, \mathbb{R})$ треугольных матриц $g=$ $\left(\begin{array}{cc}\epsilon^{-1} & 0 \\ \gamma & \epsilon\end{array}\right)$ записывать в виде пар $(\epsilon, \gamma)$ с законом умножения $\left(\epsilon_{1}, \gamma_{1}\right)\left(\epsilon_{2}, \gamma_{2}\right)=$ $\left(\epsilon_{1} \epsilon_{2}, \gamma_{1} \epsilon_{2}^{-1}+\epsilon_{1} \gamma_{2}\right)$. Группа $P$ есть полупрямое произведение $\mathbb{R}_{+}^{*} \wedge P_{0}$, где $P_{0}-$ подгруппа группы $P$ элементов, у которых $\epsilon= \pm 1$.

Имеются, с точностью до перехода к сопряженным представлениям, два унитарных неприводимых канонических представления $T^{ \pm}$группы $P_{0}$ в одномерных пространствах $H^{ \pm}$и одно ортогональное представление $T^{0}$ в двумерном пространстве $H^{0}$. Операторы представлений $T_{r}^{ \pm}$действуют в пространствах $H_{r}^{ \pm}=H^{ \pm}$по формулам $T_{r}^{ \pm}(1, \gamma)=e^{ \pm i r^{2} \gamma} \mathrm{id}, T_{r}^{ \pm}( \pm 1,0)=\mathrm{id}$. Соответственно счетные тензорные произведения $H_{\xi}^{ \pm}, \xi=\left\{r_{k}, x_{k}\right\}$, на которых действует группа токов $P_{0}^{X}$, также одномерны, а потому пространствами INT $H^{ \pm}$представлений $U^{ \pm}=\operatorname{INT} T^{ \pm}$группы $P^{X}$ являются гильбертовы пространства комплекснозначных функционалов $F^{ \pm}(\xi)=F\left(\left\{r_{k}, x_{k}\right\}\right)$ на $l_{+}^{1}(X)$ с нормой

$$
\|F\|^{2}=\int_{l_{+}^{1}(X)}|F(\xi)|^{2} d \mathscr{L}(\xi) .
$$


Операторы представления группы $P^{X}$ тождественны на ее центре и однозначно определяются формулами

$$
\begin{aligned}
& \left(U^{ \pm}(1, \gamma(\cdot)) F^{ \pm}\right)(\xi)=e^{ \pm i \sum r_{k}^{2} \gamma\left(x_{k}\right)} F^{ \pm}(\xi), \\
& \left(U^{ \pm}\left(r_{0}(\cdot), 0\right) F\right)(\xi)=e^{\frac{1}{2} \int_{X} \log r_{0}(x) d m(x)} F^{ \pm}\left(r_{0}(\cdot) \xi\right) .
\end{aligned}
$$

Операторы ортогональных представлений $T_{r}^{0}$ реализуются в двумерных вещественных подпространствах $H_{r}^{0} \subset H_{r}^{+} \oplus H_{r}^{-}$векторов вида $(f, \bar{f})$ и являются ограничениями на эти подпространства операторов $T_{r}^{+}(g) \oplus T_{r}^{-}(g)$. Поэтому пространства $H_{\xi}^{0}$ представлений группы $P_{0}^{X}$ являются счетными тензорными произведениями двумерных вещественных пространств, и интегральная модель $U^{0}=\mathrm{INT} T^{0}$ представления группы $P^{X}$ действует в прямом интеграле по мере $\mathscr{L}$ этих пространств INT $H^{0}=\int_{l_{+}^{1}(X)} H_{\xi}^{0} d \mathscr{L}(\xi)$. Операторы $\left(U^{0}\left(r_{0}(\cdot), 0\right)\right)$ этого представления задаются той же формулой $(6)$, а операторы $U^{0}(1, \gamma(\cdot))$ формулой

$$
U^{0}(1, \gamma(\cdot))\left(\bigotimes\left(f_{k}, \bar{f}_{k}\right)\right)=\bigotimes_{k=1}^{\infty}\left(e^{i r_{k}^{2} \gamma\left(x_{k}\right)} f_{k}, e^{-i r_{k}^{2} \gamma\left(x_{k}\right)} \bar{f}_{k}\right)
$$

Заметим, что при естественном вложении $\operatorname{INT} H^{0} \subset \operatorname{INT}\left(H^{+} \oplus H^{-}\right)$операторы $U^{0}(g)$ являются ограничениями на INT $H^{0}$ операторов $U(g)$ в пространстве $\operatorname{INT}\left(H^{+} \oplus H^{-}\right)$.

Опишем продолжения представлений $U^{ \pm}$и $U^{0}$ группы токов $P^{X}$ на группу $S L(2, \mathbb{R})^{X}$.

Введем однородное пространство группы $G=S L(2, \mathbb{R})$ - верхнюю комплексную полуплоскость $L$ с заданным на ней действием группы $G: z \mapsto g z=\frac{\delta z+\gamma}{\beta z+\alpha}$ при $g=\left(\begin{array}{ll}\alpha & \beta \\ \gamma & \delta\end{array}\right)$ (плоскость Лобачевского). Обозначим через $L^{X}$ пространство ограниченных функций $z: X \rightarrow L$. Отметим, что в силу ограниченности интеграл $\int_{X} \log |z(x)| d m(x)$ сходится для любой функции $z \in L^{X}$. Действие группы $G$ на $L$ индуцирует поточечное действие группы $G^{X}$ на $L^{X}$.

Введем далее функцию

$$
c\left(z_{1}, z_{2}\right)=\log \left(-i\left(z_{1}-\overline{z_{2}}\right)\right)=\log \left[\left(v_{1}+v_{2}\right)-i\left(u_{1}-u_{2}\right)\right]
$$

на $L \times L$, где $z_{k}=u_{k}+i v_{k}$, и определим функции $u^{ \pm}(g, z)$ на $G \times L$ равенствами

$$
u^{+}(g, z)=\overline{u^{-}(g, z)}=c\left(g z, g z_{0}\right)-c\left(z, z_{0}\right)-\frac{1}{2}\left(c\left(g z_{0}, g z_{0}\right)-c\left(z_{0}, z_{0}\right)\right),
$$

где $z_{0}=i$.

Для продолжения представлений $U^{ \pm}=\operatorname{INT} T^{ \pm}$группы $P^{X}$ на $S L(2, \mathbb{R})^{X}$ поставим в соответствие каждой функции $z \in L^{X}$ следующие функционалы на $l_{+}^{1}(X)$ :

$$
F_{z}^{+}(\xi)=e^{i \sum r_{k}^{2} z\left(x_{k}\right)}, \quad F_{z}^{-}(\xi)=\overline{F_{z}^{+}(\xi)}=e^{-i \sum r_{k}^{2} \overline{z\left(x_{k}\right)}}
$$

Эти функционалы принадлежат пространствам INT $H^{ \pm}$и образуют в них тотальные подмножества. 
Определим действие операторов $U^{ \pm}(g)$ группы токов $S L(2, \mathbb{R})^{X}$ на множествах функционалов $F_{z}^{ \pm}$следующей формулой:

$$
U^{ \pm}(g) F_{z}^{ \pm}=e^{\frac{1}{2} \int_{X} u^{ \pm}(g(x), z(x)) d m(x)} F_{g z}^{ \pm},
$$

где $u^{ \pm}(g, z)$ задается равенством $(9)$.

Теорема 4. Oператоры $U^{ \pm}(\cdot)$, задаваемъе формулой (10), продолэсаются до унитарных операторов на соответствующих пространствах $\mathrm{INT}^{ \pm} u$ задают там проективные представления группь $G^{X}$; ограничения этих представлений на подгруппу $P^{X}$ совпадают с исходными представлениями этой nодаруппь.

Для продолжения ортогонального представления $U^{0}=\operatorname{INT} T^{0}$ группы $P^{X}$ в пространстве INT $H^{0}$ на группу $S L(2, \mathbb{R})^{X}$ поставим в соответствие каждой функции $z \in L^{X}$ следующий функционал на $l_{+}^{1}(X)$ :

$$
F_{z}^{0}(\xi)=\bigotimes_{k=1}^{\infty}\left(2^{-1 / 2} e^{i r_{k}^{2} z\left(x_{k}\right)}, 2^{-1 / 2} e^{-i r_{k}^{2} \overline{z\left(x_{k}\right)}}\right) .
$$

Функционалы $F_{z}^{0}$ принадлежат пространству INT $H^{0}$ и образуют в нем тотальное подмножество.

Определим операторы $U^{0}(g)$ группы $P^{X}$ на множестве функционалов $F_{z}^{0}$ равенством

$$
U^{0}(g) F_{z}^{0}=e^{\frac{1}{2} \operatorname{Re} \int_{X} u^{ \pm}(g(x), z(x)) d m(x)} F_{g z}^{0},
$$

где $u^{ \pm}(g, z)$ по-прежнему задается формулой $(9)$.

Теорема 5. Oператорь $U^{0}(g)$ продолжсаются до ортогональных операторов на INT $H^{0}$ и задают на INT $H^{0}$ ортогональное представление группь $G^{X}$, ограничение которого на подгруппу $P^{X}$ совпадает с исходным. Полученное представление группъ $S L(2, \mathbb{R})^{X}$ эквивалентно представлению, построенномy в [2].

\section{§6. Продолжение интегральных моделей представлений максимальных параболических подгрупп групп $O(n, 1)$ и $U(n, 1)$ до представлений этих групп}

Известно (см., например, [8]), что группы $O(n, 1)$ и $U(n, 1)$ обладают единственным ортогональным неприводимым особым представлением. Его ограничение на максимальную параболическую подгруппу $P$ также неприводимо и ассоциировано с каноническим ортогональным представлением подгруппы $P_{0}$, входящей в разложение $P=\mathbb{R}_{+}^{*} \wedge P_{0}$; обозначим через $T^{0}$ это каноническое ортогональное представление. Возникает вопрос о возможности продолжения ортогонального представления INT $T^{0}$ группы $P^{X}$ до представлений групп $O(n, 1)^{X}$ и $U(n, 1)^{X}$.

Теорема 6. Интегральные модели INT $T^{0}$ ортогональных представлений групп $P^{X}$, где $P \subset O(n, 1)$ или $P \subset U(n, 1)$, допускают продолжение до ортогональных (непроективных) представлений групп $O(n, 1)^{X}$ u $U(n, 1)^{X}$, эквивалентных их фоковским ортогональным представлениям, описанным в [5]. Сплетающий оператор этих ортогональных представлений групп $O(n, 1)^{X} u$ $U(n, 1)^{X}$ порожден отображением соответствующих ииклических векторов.

Утверждение следует из совпадения сферических функций соответствующих представлений группы $P^{X}$. 
В случае группы $U(n, 1)$ в ее особом ортогональном неприводимом представлении можно двумя способами ввести структуру унитарного представления. Ограничения полученных таким образом особых унитарных представлений группы $U(n, 1)$ на подгруппу $P$ неприводимы и ассоциированы с каноническими унитарными представлениями $T^{+}$и $T^{-}$подгруппы $P_{0}$.

Теорема 7. Интегральные модели $\mathrm{INT}^{ \pm}$унитарных представлений группы $P^{X}$, ассоииированных с каноническими унитарными представлениями $T^{ \pm}$ подгруппы $P_{0}$, продолжсаемы до проективных унитарных представлений группь токов $U(n, 1)^{X}$, проективно эквивалентных ее боковским проективным унитарным представлениям, описанным в [6].

Полученные так продолжения являются новыми моделями представлений групп токов $O(n, 1)^{X}$ и $U(n, 1)^{X}$, в которых пространство представления реализовано как прямой интеграл по мере $\mathscr{L}$ подпространств, инвариантных относительно подгруппы $P_{0}^{X}$, а элементы из $\left(\mathbb{R}_{+}^{*}\right)^{X} \subset P^{X}$ переставляют эти подпространства.

Приведем явное описание интегральных моделей $\operatorname{INT}^{ \pm}$и $\operatorname{INT}^{0}$ для случая групп $P \subset U(n, 1)$ и их продолжений до представлений групп токов $U(n, 1)^{X}$.

Реализуем $U(n, 1)$ как группу линейных преобразований в $\mathbb{C}^{n+1}$, сохраняющих эрмитову форму $x_{1} \bar{x}_{n+1}+x_{n+1} \bar{x}_{1}+\left|x_{2}\right|^{2}+\cdots+\left|x_{n}\right|^{2}$, и будем представлять элементы $g \in U(n, 1)$ в блочной форме: $g=\left\|g_{i j}\right\|_{i, j=1,2,3}$, где на диагонали стоят матрицы порядков $1, n-1$ и 1 соответственно. В этой реализации $P$ есть подгруппа в $U(n, 1)$ всех нижних треугольных клеточных матриц. Она представима в виде полупрямого произведения: $P=\mathbb{R}_{+}^{*} \wedge P_{0}$, где $P_{0}=D_{0} \wedge N, N$ - группа Гейзенберга порядка $2 n-1$, реализованная как группа пар $(t, z) \in \mathbb{R} \otimes \mathbb{C}^{n-1}$, и $D_{0} \cong U(1) \times U(n-1)$.

В конструкции интегральных моделей INT $T^{ \pm}$и $\operatorname{INT}^{0}$ канонические унитарные представления $T^{ \pm}$группы $P_{0}$ реализуются в пространствах $H^{ \pm}$соответственно целых аналитических и целых антианалитических функций $f(z)$ на $\mathbb{C}^{n-1}$ с нормой $\|f\|^{2}=\int_{\mathbb{C}^{n-1}}|f(z)|^{2} e^{-z z^{*}} d \mu(z)$ (относительно действия $P_{0}$ на $H^{ \pm}$см. [8]). Ортогональное каноническое представление $T^{0}$ реализуется в подпространстве $H^{0} \subset H^{+} \oplus H^{-}$векторов вида $(f, \bar{f})$.

В соответствии с общим определением представления $U^{ \pm}=\mathrm{UNT}^{ \pm}$и $U^{0}=\mathrm{UNT}^{0}$ группы $P^{X}$ реализуются соответственно в пространствах INT $H^{ \pm}=\int_{l_{+}^{1}(X)}^{\oplus} H_{\xi}^{ \pm} d \mathscr{L}(\xi)$, где $H_{\xi}^{ \pm}=\bigotimes_{k=1}^{\infty} H_{r_{k}}^{ \pm}$, и в пространстве INT $H^{0}=$ $\int_{l_{+}^{1}(X)}^{\oplus} H_{\xi}^{0} d \mathscr{L}(\xi)$, где $H_{\xi}^{0}=\bigotimes_{k=1}^{\infty} H_{r_{k}}^{ \pm}$.

Конструкция их продолжений на всю группу $U(n, 1)^{X}$ аналогична конструкции продолжений для случая $S L(2, \mathbb{R})$. А именно, вместо плоскости Лобачевского здесь вводится однородное пространство $L$ группы $U(n, 1)$, эквивалентное диску в $\mathbb{C}^{n}$ ( $n$-мерное комплексное пространство Лобачевского),

$$
L=\left\{v=(a, b) \in \mathbb{C} \oplus \mathbb{C}^{n-1} \mid a+\bar{a}+b^{*} b<0\right\}, \quad \text { где } b^{*} b=\sum \bar{b}_{i} b_{i}
$$

$(b$ - вектор-столбец). Действие группы $U(n, 1)$ на $L$ задается формулой $g(a, b)=$ $\left(a^{\prime}, b^{\prime}\right)$, где

$$
\begin{aligned}
& a^{\prime}=\left(g_{11}+g_{12} b+g_{13} a\right)^{-1}\left(g_{31}+g_{32} b+g_{33} a\right), \\
& b^{\prime}=\left(g_{11}+g_{12} b+g_{13} a\right)^{-1}\left(g_{21}+g_{22} b+g_{23} a\right) .
\end{aligned}
$$


Введем на $L \times L$ функцию

$$
c\left(v_{1}, v_{2}\right)=\log \left(-a_{1}-\bar{a}_{2}-b_{2}^{*} b_{1}\right) \quad \text { при } v_{i}=\left(a_{i}, b_{i}\right) \in L .
$$

Обозначим далее через $L^{X}$ множество измеримых ограниченных отображений $v: X \rightarrow L$ с заданным на нем поточечным действием $v \mapsto g v$ группы $P^{X}$. Отметим, что из условия ограниченности функций $v \in L^{X}$ следует сходимость интегралов $\int_{X} c(v(x), v(x)) d m(x)$ для всех $v \in L^{X}$.

Свяжем с каждой тройкой $v=(a, b) \in L^{X}, r \in \mathbb{R}_{+}^{*}$ и $x \in X$ вектор $f_{v, r, x} \in H_{r}$,

$$
f_{v, r, x}(z)=\exp \left(r^{2} a(x)+r(z, b(x))\right), \quad(z, b)=\sum z_{i} b_{i},
$$

и поставим в соответствие элементам $v \in L^{X}$ следующие функционалы $F_{v}^{ \pm}(\xi)$ и $F_{v}^{0}(\xi)$ на $l_{+}^{1}(X)$ :

$$
F_{v}^{+}(\xi)=\overline{F_{v}^{-}(\xi)}=\bigotimes_{k=1}^{\infty} f_{v, r_{k}, x_{k}}, \quad F_{v}^{0}(\xi)=\bigotimes_{k=1}^{\infty}\left(2^{-1 / 2} f_{v, r_{k}, x_{k}}, 2^{-1 / 2} \overline{f_{v, r_{k}, x_{k}}}\right) .
$$

Эти функционалы принадлежат соответственно пространствам INT $H^{ \pm}$и INT $H^{0}$ и образуют в них тотальные подмножества.

Определим на этих множествах действия операторов $U^{ \pm}(g)$ и $U^{0}(g)$ теми же формулами $(10)$ и $(11)$, что и в случае группы $S L(2, \mathbb{R})$, в которых следует лишь заменить $z$ на $v$, функцию $c\left(z_{1}, z_{2}\right)$ на введенную здесь функцию $c\left(v_{1}, v_{2}\right)$ и вместо $z_{0}=i$ писать $v_{0}=(-1,0)$.

Теорема 8. Операторы $U^{ \pm}(g)$ и $U^{0}(g)$ продолжсаются до унитарных и ортогонального операторов в пространствах INT $H^{ \pm} u$ INT $H^{0}$ соответственно и задают продолжение исходных представлений группы $P^{X}$ до проективных унитарных и ортогонального представлений группы $U(n, 1)^{X}$ соответственно.

\section{ЛитеРАтУРА}

[1] H. Araki, Factorizable representations of the current algebra, Publ. RIMS Kyoto Univ. Ser. A, 5:3 (1970), 361-422.

[2] А. М. Вершик, И. М. Гельфанд, М. И. Граев, Представления группы $S L(2, R)$, где $R$ - кольи, функиий, УМН, 28:5 (1973), 83-128.

[3] А. М. Вершик, И. М. Гельфанд, М. И. Граев, Неприводимые представления груп$n ъ G^{X}$ и когомологии, Функц. анализ и его прил., 8:2 (1974), 67-69.

[4] Ф. А. Березин, Представления непрерывного прямого произведения универсальных накрывающих группы движений комплексного шара, Труды ММО, 36 (1978), $275-293$.

[5] I. M. Gelfand, M. I. Graev, A. M. Vershik, Models of representations of current groups, in: Representations of Lie groups and Lie algebras, Akad. Kiado, Budapest, 1985, 121179 .

[6] И. М. Гельфанд, М. И. Граев, Особые представления группы $S U(n, 1)$ и проективные унитарные представления группы токов $S U\left(n, 1^{X}\right)$, Докл. РАН, 332:3 (1993), 280-282.

[7] А. М. Вершик, М. И. Граев, Коммутативная модель представления группы $O(n, 1)^{X}$ и обобщенная лебегова мера в пространстве распределений, Функц. анализ и его прил., 39:2 (2005), 1-12.

[8] А. М. Вершик, М. И. Граев, Структура дополнительных серий и особых представлений групп $O(n, 1)$ и $U(n, 1)$, УМH, 61:5 (2006), 1-88. 
[9] M. I. Graev, A. M. Vershik, The basic representation of the current group $O(n, 1)^{X}$ in the $L^{2}$ space over the generalized Lebesgue measure, Indag. Math., 16:3/4 (2005), 499-529.

[10] А. М. Вершик, М. И. Граев, Интегральные модели представлений групп токов, Функц. анализ и его прил., 42:1 (2008), 22-32.

[11] А. М. Вершик, Существует ли мера Лебега в бесконечномерном пространстве?, Труды МИРАН, 259 (2007).

[12] A. Vershik, Invariant measures for continual Cartan subgroup, J. Funct. Anal., 2008 (в печати).

[13] А. М. Вершик, С. И. Карпушев, Когомологии групп в унитарных представлениях, окрестность единицы и условно положительно определенные функиии, Матем. сб., 119:4 (1982), 521-533.

[14] А. М. Вершик, Н. В. Цилевич, Фоковские факторизации и разложения пространства $L^{2}$ над общими процессами Леви, УМН, 58:3 (351) (2003), 3-50.

[15] N. Tsilevich, A. Vershik, M. Yor, An infinite-dimensional analogue of the Lebesgue measure and distinguished properties of the gamma process, J. Funct. Anal., 185:1 (2001), 274-296.

[16] R. S. Ismagilov, Representations of Infinite-Dimensional Groups, Transl. Math. Monograph., vol. 152, Amer. Math. Soc., Providence, RI, 1996.

[17] B. Tsirelson, A. Vershik, Examples of nonlinear continuous tensor products of measure spaces and non-Fock factorizations, Rev. Math. Phys., 10:1 (1998), 81-145.

[18] Э. Хьюитт, К. Росс, Абстрактный гармонический анализ, т. 2, М., Мир, 1970.

С.-Петербургское отделение Математического

института им. В. А. Стеклова

Поступило в редакцию

e-mail: vershik@pdmi.ras.ru

Институт системных исследований РАН, Москва

e-mail: graev_36@mtu-net.ru

11 августа 2008 г. 